\title{
Healthcare Workers' Knowledge and Attitudes Regarding the World Health Organization's "My 5 Moments for Hand Hygiene": Evidence From a Vietnamese Central General Hospital
}

\author{
Huy Van Nguyen ${ }^{1,2,3 *}$, Hieu Trung Tran ${ }^{2 *}$, Long Quynh Khuong ${ }^{4}$, Thanh Van Nguyen ${ }^{2}$, Na Thi Nhi Ho ${ }^{5}$, An Thi Minh Dao ${ }^{2,3}$, \\ Minh Van Hoang ${ }^{6}$ \\ 'Graduate School of Public Health, St. Luke's International University, Tokyo, Japan; ' Institute for Preventive Medicine and Public Health, Hanoi \\ Medical University, Hanoi, Vietnam; ${ }^{3}$ Department of Population and Quantitative Health Sciences at the University of Massachusetts Medical \\ School, Worcester, MA, USA; ${ }^{4}$ Center for Population Health Science, Hanoi University of Public Health, Vietnam; ${ }^{5}$ School of Nursing, Duy Tan \\ University, Da Nang, Vietnam; ${ }^{6}$ Department of Health Economics, Hanoi University of Public Health, Hanoi, Vietnam
}

Objectives: Although the World Health Organization (WHO) initiative "My 5 Moments for Hand Hygiene" has been lauded as effective in preventing hospital-associated infections, little is known about healthcare workers (HCWs)' hand hygiene behavior. In this study, we sought to assess knowledge and attitudes towards the concepts in this initiative, as well as associated factors, among Vietnamese HCWs at a general hospital.

Methods: A structured questionnaire was administered to HCWs at a central Vietnamese general hospital in 2015. Multiple logistic regression analysis was used to identify factors associated with $\mathrm{HCWs}$ ' knowledge and attitudes towards hand hygiene.

Results: Of 120 respondents, $65.8 \%$ and $67.5 \%$ demonstrated appropriate knowledge and a positive attitude, respectively, regarding all 5 hand hygiene moments. Logistic regression indicated better knowledge of hand hygiene in workers who were over 30 years old, who were direct HCWs (rather than managers), who had frequent access to clinical information, and who received their clinical information from training. Those who worked in infectious and tropical disease wards, who had frequent access to clinical information, and who received information from training were more likely to have a positive attitude towards hand hygiene than their counterparts.

Conclusions: Although many Vietnamese HCWs displayed moderate knowledge and positive attitudes towards the WHO hand hygiene guidelines, a key gap remained. Regular education and training programs are needed to increase knowledge and to improve attitudes and practices towards hand hygiene. Furthermore, a combination of multimodal strategies and locally-adapted interventions is needed for sustainable hand hygiene adherence.

Key words: Knowledge, Attitude, Hand hygiene, Health workers, World Health Organization, Vietnam

Received: November 25, 2019 Accepted: April 9, 2020

Corresponding author: Huy Van Nguyen, MD, PhD Graduate School of Public Health, St. Luke's International University,

3-6-2 Tsukiji, Chuo-ku, Tokyo 104-0045, Japan

E-mail: nguyenhuy@sicn.ac.jp

*Huy and Hieu contributed equally to this work as joint first authors.

This is an Open Access article distributed under the terms of the Creative Commons Attribution Non-Commercial License (https://creativecommons.org/licenses/by$\mathrm{nc} / 4.0 /$ ) which permits unrestricted non-commercial use, distribution, and reproduction in any medium, provided the original work is properly cited.

\section{INTRODUCTION}

Hospital-associated infections (HAls) are a global issue and cause millions of deaths annually. These infections are frequent concerns at every healthcare facility and severely impact millions of patients worldwide [1]. HAls lengthen hospital stays, increase the financial burden for patients and hospitals, promote the antibiotic resistance of microorganisms due 
to associated treatment, and contribute to mortality [1]. However, HAls are preventable, and compliance with standard precautions is one of the most effective practices to prevent HAls [2].

Hand hygiene is the most feasible and affordable of these standard precautions. It has been reported that HAls could be reduced by $15 \%$ to $30 \%$ if healthcare workers (HCWs) regularly complied with recommended hand hygiene practices [3]. The World Health Organization (WHO) recommendations regarding 5 crucial moments of hand hygiene have been successfully implemented in healthcare facilities worldwide [1]. However, despite the critical role of hand hygiene, numerous recent findings have revealed low compliance with related guidelines in various healthcare settings. A study in Australia determined that only $68.3 \%$ of the HCWs studied demonstrated overall hand hygiene compliance [4]. In another study from 2013, Allegranzi et al. [5] reported this rate as 51.0\% among HCWs in 5 countries before intervention. Hand hygiene compliance in Thailand, Uganda, and Mali was found to range widely from $8.0 \%$ to $74.7 \%$, indicating that the implementation of these practices has not been sufficient in developing countries [6-8].

Vietnam is facing a similar situation regarding adherence to patient safety programs [9]. Although a previous study showed that the majority of Vietnamese HCWs displayed adequate knowledge and positive attitudes $(79.1 \%$ and $70.0 \%$, respectively), the score for actual practice regarding hospital standard precautions was lower, at $46.1 \%$ [10]; as a result, the average rate of HAls in 36 Vietnamese hospitals was $7.8 \%$ among 7571 admitted patients [11]. More recent evidence suggests that this rate may be even higher (31.7\%) [12]. A recent report indicated that hand hygiene compliance among Vietnamese HCWs was only $47.0 \%$ and varied across clinical departments [13], although the WHO guidelines for improving and promoting hand hygiene have been officially endorsed for clinical practice by the Vietnam Ministry of Health since 2012 [14]. However, since then, little evaluation has been performed.

A high rate of non-adherence to hand hygiene practices among HCWs could be influenced by many factors, including lack of education, lack of awareness, facilities, and environment $[3,15,16]$. A few studies have pointed to some noteworthy obstacles in the practice of hand hygiene in Vietnam, such as limited essential resources and overcrowded healthcare settings [17]. A successful attempt was made to improve the hand hygiene compliance rate ( $25.7 \%$ before the intervention to $57.5 \%$ after the intervention) [12]. However, previous studies in this field have appeared to focus on hand hygiene compliance and did not address the existence of adequate knowledge and positive attitudes towards WHO hand hygiene guidelines among Vietnamese HCWs. The objective of this paper was to investigate whether a shortage of knowledge and positive attitudes existed regarding the WHO's "My 5 Moments for Hand Hygiene" guidelines among HCWs in a Vietnamese general hospital and to assess associated factors.

\section{METHODS}

\section{Design and Setting}

This cross-sectional study was conducted at Quang Nam Central General Hospital, which is a general hospital located in central Vietnam. Central (or national) hospitals comprise the top level of the 4-level hierarchy of the Vietnamese healthcare system (national, provincial, district, and communal hospitals). Established in 2007 with financial support largely from the Korea, Quang Nam Central General Hospital is a 600-bed healthcare facility serving the populations of Quang Nam Province and surrounding areas. As a leading general hospital in this region, it is equipped with modern facilities and medical technology to diagnose and provide high-quality treatment to meet local healthcare needs. The hospital has 24 clinical and subclinical wards and serves approximately 600 inpatients and 800 outpatients daily. Under the hand hygiene guidelines from both the WHO and the Vietnam Ministry of Health, all Vietnamese hospitals are required to take action on hand hygiene.

\section{Participants and Sample}

All HCWs contracted to work for at least 6 months in 1 of 4 clinical wards (general surgery, obstetrics and gynecology, trauma and orthopedics, and infectious and tropical disease) were recruited because they had long-term contracts and were therefore categorized as official HCWs according to Vietnamese law [18]. Those contracted to work for less than 6 months were instead categorized as temporary or probationary employees. A total of 120 respondents were eligible and participated in the study, yielding a response rate of $100 \%$. We strategically selected these wards, as they treat a high number of inpatients and are subject to numerous standard precautionary procedures. 


\section{Instrument and Measures}

The questionnaire in this survey was designed mainly based on the WHO "My 5 Moments for Hand Hygiene" concept [1] and the Vietnamese Ministry of Health guidelines on hand hygiene, which were also adapted from the WHO guidelines on hand hygiene in healthcare [14]. The survey used in the present study had 3 components: demographic questions, a test of hand hygiene knowledge, and a scale representing the respondent's attitude towards hand hygiene. The demographic data focused on age, sex, professional role, work position, clinical ward, years of experience, main source of access to clinical information, and frequency of access to clinical information (with frequent access defined as the receipt of relevant information at least once a month). All of these data, except for age and years of experience, were collected via a series of closed-ended questions with a list of provided responses. The knowledge and attitude of HCWs regarding hand hygiene were measured in the context of 5 situations: before contact with a patient, before an aseptic procedure, after contact with a patient, after contact with body fluid, and after contact with patient surroundings. The hand hygiene knowledge instrument consisted of 19 true-false statements about the 5 moments of hand hygiene. A correct answer to a particular true-false statement was coded as 1 and an incorrect answer as 0 . The assessment of hand hygiene attitude included 9 items regarding the 5 moments of hand hygiene answered on a 5 -point Likert scale (1, strongly disagree; 2 , disagree; 3 , uncertain; 4 , agree; 5 , strongly agree), with a higher score reflecting a more positive attitude. Composite scores were obtained by summing correct knowledge and positive attitude responses, with higher scores indicating better knowledge of and a more positive attitude towards hand hygiene, respectively. A participant's knowledge of hand hygiene was classified as good when the participant correctly answered all of the items on the knowledge questionnaire. Similarly, any participant who chose 4 (agree) or 5 (strongly agree) for all questions in the attitude section was considered to have a positive attitude towards hand hygiene overall. As no guidelines were available in the literature regarding cut-off values for hand hygiene, we required all answers to be correct and all answers to indicate positive attitude in the assessments of knowledge and attitude, respectively. The knowledge and attitude questions were carefully separated to avoid priming. Since the knowledge and attitude assessments related to the procedures recommended for HCWs in general, the instrument and scoring criteria were applied in the same manner for both physicians and nurses. The quality, clarity, and feasibility of the questionnaire were evaluated via a pilot study conducted among 20 HCWs. The pilot showed that the questionnaire was technically feasible for the main survey. The reliability of the knowledge and attitude questionnaires for the main survey was confirmed by Cronbach alpha values of 0.71 and 0.67 , respectively.

\section{Data Collection}

Data collection was conducted by well-trained researchers. Before the survey, these researchers were familiarized with relevant aspects of the research, survey and sampling methods, the selection of study subjects, and the proper handling of missing data from subjects. Then, they interviewed respondents via a structured questionnaire. Each participant was interviewed at his or her workplace. We chose to conduct faceto-face interviews to minimize missing data as well as to help collect valid responses. The data collection took place over 3 months, from August to November 2015. One of our principal investigators was always present in the field as a supervisor to support the data collection process.

\section{Statistical Analysis}

All data were collected in written format and were then entered using EpiData version 3.1 (EpiData Association, Odense, Denmark). All analyses were performed using Stata version 12.1 (StataCorp., College Station, TX, USA). Descriptive statistics were used for the questions regarding knowledge and attitude, while multivariable logistic regression was employed to build models of factors associated with appropriate knowledge of, and positive attitude towards, the WHO 5 moments of hand hygiene, with $p$-value $<0.05$ indicating statistical significance. The reliability of the questionnaires was analyzed using Cronbach alpha values to assess internal consistency, as presented above. For logistic regression models with adequate sample sizes, composite scores of knowledge and attitude were categorized using the midpoint as a cut-off to serve as binary dependent variables. Age, sex, professional role, work position, clinical ward, years of experience, frequency of access to clinical information, and clinical information sources were examined as predictors in the models. The model fit was assessed with the Hosmer-Lemeshow goodness-of-fit test (with a $p$-value for model fit from the chi-square test $>0.05, p$-values of model coefficients $<0.05$, and a high value for the Nagelkerke 
$R^{2}$ ). The final models for knowledge and attitude included only those variables that were found to be significant.

\section{Ethics Statement}

The study protocol was approved by the Scientific Panel of the Institute for Preventive Medicine and Public Health at Hanoi Medical University according to Decision No. 61/QDYHDP\&YTCC, dated $16 / 06 / 2015$. The objectives of the study were explained to participants, and they chose to participate in the study voluntarily, after providing informed consent. Data confidentiality was maintained at all phases of the survey, and results were reported in aggregate rather than being

Table 1. Demographic characteristics of respondents $(n=120)$

\begin{tabular}{|c|c|}
\hline Characteristics & $n(\%)$ \\
\hline \multicolumn{2}{|l|}{ Age (y) } \\
\hline$<30$ & $61(50.8)$ \\
\hline$\geq 30$ & $59(49.2)$ \\
\hline \multicolumn{2}{|l|}{ Sex } \\
\hline Female & $94(78.3)$ \\
\hline Male & $26(21.7)$ \\
\hline \multicolumn{2}{|l|}{ Professional role } \\
\hline Clinician & $21(17.5)$ \\
\hline Nurse & $99(82.5)$ \\
\hline \multicolumn{2}{|l|}{ Work position } \\
\hline Manager $^{2}$ & $12(10.0)$ \\
\hline Healthcare worker & $108(90.0)$ \\
\hline \multicolumn{2}{|l|}{ Clinical ward } \\
\hline Obstetrics and gynecology & $48(40.0)$ \\
\hline Trauma and orthopedics & $28(23.3)$ \\
\hline General surgery & $26(21.7)$ \\
\hline Infectious and tropical disease & $18(15.0)$ \\
\hline \multicolumn{2}{|l|}{ Years of experience $(y)^{3}$} \\
\hline$<5$ & $47(39.2)$ \\
\hline $5-10$ & $50(41.7)$ \\
\hline $10-20$ & $13(10.8)$ \\
\hline$>20$ & $10(8.3)$ \\
\hline \multicolumn{2}{|l|}{ Frequent access to clinical information (via various sources) } \\
\hline No & $28(23.3)$ \\
\hline Yes & $92(76.6)$ \\
\hline \multicolumn{2}{|l|}{ Main source of access to clinical information } \\
\hline Mass media (Internet, medical journals, television, radio) & $10(8.5)$ \\
\hline Training & $107(91.5)$ \\
\hline
\end{tabular}

${ }^{1}$ Clinicians: physicians from 4 clinical wards.

${ }^{2}$ Managers: heads and/or vice heads of the wards who play both doctorial and managerial roles.

${ }^{3}$ In Vietnam, as there is no formal document defining the 5-year increments in classifying the experience of health professionals, we used this scale for ranking work seniority based on the social preference. associated with separate individuals. All collected information was kept confidential and was only used for research purposes.

\section{RESULTS}

\section{Key Characteristics of the Sample}

As seen in Table 1, among the respondents surveyed, most were female (78.3\%), and the majority were nurses (82.5\%). Approximately equivalent numbers of respondents were younger than 30 years and older than 30 years (with each group accounting for about 50\%). Most of the respondents (90.0\%) were HCWs; only $10.0 \%$ were managers of clinical wards, and most had 10 years of work experience or less. Those surveyed indicated that they had frequent access to clinical information, and the primary source of clinical information was training (76.5\%).

\section{Knowledge About Hand Hygiene Among Healthcare Workers}

Results regarding knowledge about hand hygiene among HCWs are displayed in Table 2. More than $80 \%$ of the responses in each category were correct, and nearly all (90.8\%) of those surveyed knew that hand hygiene was necessary before coming into contact with patients. However, only slightly over half of participants (65.8\%) answered correctly regarding all 5 moments.

Table 2. Knowledge and attitude towards 5 moments for hand hygiene among healthcare workers $(n=120)$

\begin{tabular}{lr}
\hline Variables & $\mathbf{n}(\%)$ \\
\hline Appropriate knowledge & $109(90.8)$ \\
Before contact with patient & $105(87.5)$ \\
Before aseptic procedure & $102(85.0)$ \\
After contact with patient & $100(83.3)$ \\
After contact with body fluid & $105(87.5)$ \\
After contact with patient surroundings & $79(65.8)$ \\
Correct knowledge regarding all 5 moments for hand hygiene & \\
Positive attitude & $97(80.8)$ \\
Necessity of hand hygiene before contact with patient & $119(98.3)$ \\
Necessity of hand hygiene before aseptic procedure & $117(98.3)$ \\
Necessity of hand hygiene after contact with patient & $106(88.3)$ \\
Necessity of hand hygiene after contact with body fluid & $116(96.6)$ \\
Necessity of hand hygiene after contact with patient & \\
$\quad$ surroundings & $81(67.5)$ \\
\hline Attitude that all 5 moments for hand hygiene are necessary &
\end{tabular}




\section{Attitude Towards Hand Hygiene Among} Healthcare Workers

Results regarding HCWs' attitude towards hand hygiene are presented in Table 2. Most of the responses in each category indicated a positive attitude, ranging from $80.8 \%$ to $96.6 \%$. However, the proportion of respondents who had

Table 3. Multivariable logistic regression of factors associated with good knowledge and positive attitude regarding hygiene among healthcare workers $(n=120)$

\begin{tabular}{|c|c|c|}
\hline Variables & n (\%) & aOR (95\% CI) \\
\hline \multicolumn{3}{|l|}{ Appropriate knowledge } \\
\hline \multicolumn{3}{|l|}{ Age (y) } \\
\hline$<30$ & $42(68.9)$ & 1.00 (reference) \\
\hline$\geq 30$ & $45(76.3)$ & $4.42(1.18,16.55)$ \\
\hline \multicolumn{3}{|l|}{ Work position } \\
\hline Manager & $4(33.3)$ & 1.00 (reference) \\
\hline Healthcare worker & $83(76.9)$ & $11.28(1.99,63.77)$ \\
\hline \multicolumn{3}{|c|}{ Frequent access to clinical information } \\
\hline No & $65(70.7)$ & 1.00 (reference) \\
\hline Yes & $22(78.6)$ & $3.92(1.08,14.2)$ \\
\hline \multicolumn{3}{|l|}{ Clinical information sources } \\
\hline $\begin{array}{l}\text { Mass media (Internet, medical } \\
\text { journals, television, radio) }\end{array}$ & $6(60.0)$ & 1.00 (reference) \\
\hline Training & 78 (72.9) & $5.25(1.01,27.29)$ \\
\hline$\chi^{2} ; p$-value of model coefficients & \multicolumn{2}{|c|}{$26.55 ; p=0.002$} \\
\hline $\begin{array}{l}\chi^{2} ; p \text {-value (Hosmer- Lemeshow } \\
\text { goodness-of-fit test) }\end{array}$ & \multicolumn{2}{|c|}{$77.10 ; p=0.433$} \\
\hline$R^{2}$ & \multicolumn{2}{|r|}{0.19} \\
\hline \multicolumn{3}{|l|}{ Positive attitude } \\
\hline \multicolumn{3}{|l|}{ Ward } \\
\hline $\begin{array}{l}\text { Obstetrics and gynecology, } \\
\text { trauma and orthopedics, or } \\
\text { general surgery }{ }^{1}\end{array}$ & $47(65.3)$ & 1.00 (reference) \\
\hline Infectious and tropical disease & $34(70.8)$ & $6.53(1.43,29.80)$ \\
\hline \multicolumn{3}{|c|}{ Frequent access to clinical information } \\
\hline No & $16(17.9)$ & 1.00 (reference) \\
\hline Yes & 23 (82.6) & $20.53(7.57,29.32)$ \\
\hline \multicolumn{3}{|l|}{ Clinical information sources } \\
\hline $\begin{array}{l}\text { Mass media (Internet, medical } \\
\text { journals, television, radio) }\end{array}$ & $3(30.0)$ & 1.00 (reference) \\
\hline Training & $76(71.0)$ & $10.48(1.55,70.89)$ \\
\hline$\chi^{2} ; p$-value of model coefficients & \multicolumn{2}{|c|}{$64.30 ; p<0.001$} \\
\hline $\begin{array}{l}\chi^{2} ; p \text {-value (Hosmer-Lemeshow } \\
\text { goodness-of-fit test) }\end{array}$ & \multicolumn{2}{|c|}{$42.90 ; p=0.308$} \\
\hline$R^{2}$ & \multicolumn{2}{|r|}{0.43} \\
\hline
\end{tabular}

aOR, adjusted odds ratio; $\mathrm{Cl}$, confidence interval.

${ }^{1}$ We combined these wards due to sample size and similar clinical characteristics (these wards serve most patients suffering acute health issues and being treated mostly with surgery). positive attitudes regarding all 5 moments was surprisingly low (67.5\%).

\section{Factors Associated With Hand Hygiene Knowledge and Attitude Among Healthcare Workers}

Multivariable logistic regression was used to identify factors associated with hand hygiene knowledge among HCWs, as shown in Table 3. Knowledge of hand hygiene was significantly related to age, work position, frequency of access to clinical information, and clinical information source. HCWs who were over 30 years old, who were workers (as opposed to managers), who had frequent access to clinical information, and who received their clinical information from training were relatively likely to display appropriate knowledge of hand hygiene. The multivariable model $\left(\chi^{2}=26.55, p=0.002\right)$ explained $19.0 \%$ of the variance (Nagelkerke $R^{2}=0.19$ ). This model satisfied the Hosmer-Lemeshow goodness-of-fit standards $\left(\chi^{2}=77.10, p=\right.$ 0.433).

As shown in Table 3, attitude towards hand hygiene was found to be related to the clinical ward in which the worker was employed, frequency of access to clinical information, and clinical information source. HCWs who were employed in the infectious and tropical disease ward, who had frequent access to clinical information, and who received their clinical information from training were more likely to exhibit positive attitudes towards hand hygiene than their counterparts. The multivariable model $\left(\chi^{2}=64.30, p<0.001\right)$ explained $43.0 \%$ of the variance (Nagelkerke $R^{2}=0.43$ ). This model satisfied the HosmerLemeshow goodness-of-fit standards $\left(\chi^{2}=42.90, p=0.308\right)$.

\section{DISCUSSION}

In this study, we used a questionnaire culturally adapted from the WHO instrument "My 5 Moments for Hand Hygiene" to investigate potential gaps in knowledge and attitudes among Vietnamese HCWs regarding WHO hand hygiene guidelines.

We found that over half (65.8\%) of respondents displayed appropriate knowledge regarding all $5 \mathrm{WHO}$ moments of hand hygiene. This is similar to the findings of a study conducted in Korea, in which $68 \%$ of registered health workers exhibited high or medium levels of hand hygiene knowledge (defined as $\geq 70 \%$ of items answered correctly) [19]. The result of the present study was considerably higher than that of a previous study conducted in Iran, in which $31.2 \%$ of HCWs displayed moderate or high knowledge of hand hygiene, defined as a 
total score $\geq 50 \%$ of the maximum score [20]. However, our result was still lower than those obtained by some studies conducted in similar developing countries [15,21]. This apparent lack of concordance could be due to the measurement systems used, as different questionnaires were contextually modified from the WHO-designed Hand Hygiene Knowledge Questionnaire for Healthcare Workers. Tenna et al. [15] defined a good understanding of hand hygiene as $60 \%$ of items answered correctly on their questionnaire, while according to Ango et al. [21], those scoring above $50 \%$ on their measure were considered to have good hand hygiene knowledge. However, we believe that the adaptation of our instrument was necessary to fit the current context in Vietnam, although it may have introduced some unintended bias. Alternatively, this variation may result from different levels of progress in the quality of healthcare from country to country.

Multivariable analysis showed that age, work position, frequency of access to clinical information, and clinical information source were significantly associated with hand hygiene knowledge among HCWs. Age has been found to be a common factor across many studies. Allegranzi et al. [5], for instance, highlighted age group as a key factor positively associated with an increase in hand hygiene awareness among HCWs after intervention. Asadollahi et al. [22] reported that in Iranian nurses, age and years of experience were related to hand hygiene knowledge. However, to our knowledge, the relationship observed in this study between work position (manager vs. HCWs) and knowledge of hand hygiene has not been shown previously. One potential explanation for this relationship is that healthcare managers spend more time engaging in management than in clinical work, so they were less likely than nonmanagers to practice hand washing frequently. Moreover, as they only occasionally attended short-course training sessions, their hand hygiene knowledge was not sufficiently enhanced. The final significant factors (the frequency of access to clinical information and the individual's main clinical information source) relate to how HCWs improve their medical knowledge. Variations in the work environments and the roles of health professionals may inform how frequently they access clinical information, which contributes to their knowledge. Several studies have revealed that HCWs who previously attended more training courses on infection control were likely to have better hand hygiene knowledge than those who attended fewer such courses [8,22]. Regular training not only helps medical HCWs remain informed on infection control, but also warns them about the necessity of these measures [8]. Finally, our study did not appear to corroborate previous studies regarding relationships between HCWs' knowledge level and sex, educational background, years of experience, or professional role [5,22-24].

In terms of attitude, nearly $70 \%$ of the HCWs exhibited a moderate to positive attitude towards hand hygiene. This figure is consistent with another study conducted in Vietnam [25], but higher than those obtained in other countries $[20,26]$. Importantly, in the present study, multivariable analysis showed an association between the ward in which a HCW was employed and the likelihood of having a positive attitude. Respondents in the infectious and tropical disease ward were more likely to have a positive attitude towards hand hygiene than those in other wards. Social norms could be at play here, as many Vietnamese HCWs acknowledge the importance of hand hygiene when it is seen as relevant to their personal health and safety [17]. Also, as the infectious and tropical disease ward is at high risk for communicable diseases, HCWs are faced with dangerous pathogens on a daily basis, which is not the case for those working in other settings. Therefore, employees of infectious and tropical disease wards may display relatively high awareness in order to prevent themselves from infection. The frequency of access to clinical information and the main clinical information source also relate to attitudes among HCWs. As these factors are associated with the receipt of new medical information, they are important because they could raise HCWs' awareness of the consequences of hospital-associated infection and enhance their perceptions of patient safety.

Our findings showed that Vietnamese HCWs displayed moderate knowledge and fairly positive attitudes towards the WHO's "My 5 Moments for Hand Hygiene" as compared with data obtained elsewhere in developing countries. However, the current study did not concur with prior research on hand hygiene adherence in Vietnam, as we found higher rates of knowledge and positive attitude towards the 5 hand hygiene moments than the compliance rates reported by Thi Anh Thu et al. [12] both pre-intervention and post-intervention. The discrepancy between full understanding or positive attitude and actual practice has been mentioned in several articles focused on different settings $[15,27]$. This contradictory behavior could be explained by the theory of planned behavior, which indicates that people's actions depend not only on their knowledge, attitudes, or motivation, but also on other external influencing factors $[28,29]$. The key challenges for hand hygiene compliance among HCWs have been widely described 
as knowledge and attitude. However, these are not sufficient to strengthen hand hygiene habits [3]. Other major barriers must be considered, such as limited resources, costs, cultural issues, and constraints on feedback intended to improve hand hygiene implementation $[6,30]$. Especially in overcrowded environments (as Vietnamese healthcare settings often are), it cannot be ignored that specific challenges must also be solved [17]. To achieve a meaningful and sustainable level of hand hygiene adherence, a multimodal strategy is necessary to remove the variety of obstacles [31]. Pan et al. [32] have suggested that hand hygiene education should be highlighted in early-stage medical education aimed at raising awareness about infection control. In a successful intervention, Allegranzi et al. [5] implemented a WHO-based multimodal approach, which combined 4 components in addition to education: monitoring and feedback, visual reminders at workplaces, access to alcohol-based hand rub, and a culture that prioritized patient safety. Consistent with a systematic review, Ofek Shlomai et al. [33] indicated that providing performance feedback to HCWS at the peer, personal, or group level was relatively efficient in improving compliance. Using alcohol-based hand rub was recommended as an alternative solution for improving $\mathrm{HCWs}$ ' hand hygiene adherence in developing countries [34]. As an additional advantage, a recent study developed a modification of the WHO guidelines adapted to the local context in Vietnam, after they reported difficulties in applying the WHO's "My 5 Moments for Hand Hygiene" in such overcrowded healthcare settings [17].

Some limitations of this study exist. One methodological limitation is that we did not observe hand hygiene compliance directly; instead, due to limited budget and time, we based our results primarily on self-reported questionnaire data. Furthermore, given our cross-sectional design, it may be difficult to confirm the temporal relationship between predictors and dependent variables. Finally, as this study was conducted in a general hospital, the results might not generalize to all other hospitals in Vietnam. Despite much effort to include as many HCWs as possible in the study, the sample size remained relatively small. However, we believe that the results of our work could both reflect factual conditions at the surveyed sites and represent similar settings. Further experimental investigations are needed to determine the directions of influence.

In conclusion, this study was conducted on a sample of Vietnamese HCWs in a general hospital to assess their knowledge and attitude towards the WHO "My 5 Moments for Hand Hy- giene." Overall, our findings revealed moderate knowledge and fairly positive attitudes among HCWs compared to other studies. However, a gap remains in their knowledge and attitude that must be addressed through practical public health programs. The present study underscores the importance of regular hand hygiene training and monitoring programs to address these shortfalls. A combination of multimodal strategies and locally adapted interventions for sustainable hand hygiene adherence is likely to improve the situation further.

\section{CONFLICT OF INTEREST}

The authors have no conflicts of interest associated with the material presented in this paper.

\section{FUNDING}

None.

\section{ACKNOWLEDGEMENTS}

We appreciate the field teams for their tireless efforts to assist this research. We gratefully acknowledge the participation of all health workers in the survey in Quang Nam Central General Hospital.

\section{AUTHOR CONTRIBUTIONS}

Conceptualization: HVN. Data curation: HVN, HTT, NTNH, TVN. Formal analysis: HVN, HTT. Funding acquisition: None. Methodology: HVN, NTNH, TVN. Project administration: NTNH. Visualization: HVN, ATMD. Writing - original draft: HVN, HTT, NTNH. Writing - review \& editing: HVN, HTT, LQK, TVN, ATMD, $\mathrm{MVH}$.

\section{ORCID}

Huy Van Nguyen https://orcid.org/0000-0002-5896-9661

Hieu Trung Tran https://orcid.org/0000-0003-3293-7431

Long Quynh Khuong https://orcid.org/0000-0002-12326230

Thanh Van Nguyen $\quad$ https://orcid.org/0000-0003-3761-5706

Na Thi Nhi Ho https://orcid.org/0000-0003-3180-4725

An Thi Minh Dao https://orcid.org/0000-0002-1258-1637

Minh Van Hoang https://orcid.org/0000-0002-4749-5536 


\section{REFERENCES}

1. World Health Organization. WHO guidelines on hand hygiene in health care: a summary. First global patient safety challenge clean care is safer care; 2009 [cited 2015 Jul 1]. Available from: https://www.who.int/gpsc/5may/tools/who_guidelineshandhygiene_summary.pdf?ua $=1$.

2. Maroldi MA, Felix AM, Dias AA, Kawagoe JY, Padoveze MC, Ferreira $S A$, et al. Adherence to precautions for preventing the transmission of microorganisms in primary health care: a qualitative study. BMC Nurs 2017;16:49.

3. Huis A, van Achterberg T, de Bruin M, Grol R, Schoonhoven L, Hulscher M. A systematic review of hand hygiene improvement strategies: a behavioural approach. Implement Sci 2012;7:92.

4. Grayson ML, Russo PL, Cruickshank M, Bear JL, Gee CA, Hughes $\mathrm{CF}$, et al. Outcomes from the first 2 years of the Australian National Hand Hygiene Initiative. Med J Aust 2011;195(10):615619.

5. Allegranzi B, Gayet-Ageron A, Damani N, Bengaly L, McLaws ML, Moro ML, et al. Global implementation of WHO's multimodal strategy for improvement of hand hygiene: a quasi-experimental study. Lancet Infect Dis 2013;13(10):843-851.

6. Allegranzi B, Sax H, Bengaly L, Richet H, Minta DK, Chraiti MN, et al. Successful implementation of the World Health Organization hand hygiene improvement strategy in a referral hospital in Mali, Africa. Infect Control Hosp Epidemiol 2010;31(2): 133-141.

7. Eiamsitrakoon T, Apisarnthanarak A, Nuallaong W, Khawcharoenporn T, Mundy LM. Hand hygiene behavior: translating behavioral research into infection control practice. Infect Control Hosp Epidemiol 2013;34(11):1137-1145.

8. Wasswa P, Nalwadda CK, Buregyeya E, Gitta SN, Anguzu P, Nuwaha F. Implementation of infection control in health facilities in Arua district, Uganda: a cross-sectional study. BMC Infect Dis 2015;15:268

9. Allegranzi B, Bagheri Nejad S, Combescure C, Graafmans W, Attar $\mathrm{H}$, Donaldson $\mathrm{L}$, et al. Burden of endemic health-careassociated infection in developing countries: systematic review and meta-analysis. Lancet 2011;377(9761):228-241.

10. Thu TA, Anh NQ, Chau NQ, Hung NV. Knowledge, attitude and practices regarding standard and isolation precautions among Vietnamese health care workers: a multicenter cross-sectional survey. Intern Med Open Access 2012;2(4):115.

11. Thu TA, Hung NV, Quang NN., Archibald, LK, Thuy I, Harun-OrRashid, et al. A point-prevalence study on healthcare-associ- ated infections in Vietnam: public health implications. Infect Control Hosp Epidemiol 2011;32(10):1039-1041.

12. Thi Anh Thu L, Thi Hong Thoa V, Thi Van Trang D, Phuc Tien N, Thuy Van D, Thi Kim Anh L, et al. Cost-effectiveness of a hand hygiene program on health care-associated infections in intensive care patients at a tertiary care hospital in Vietnam. Am J Infect Control 2015:43(12):e93-e99.

13. Salmon S, Tran HL, Bùi DP, Pittet D, McLaws ML. Beginning the journey of hand hygiene compliance monitoring at a 2,100bed tertiary hospital in Vietnam. Am J Infect Control 2014; 42(1):71-73.

14. Vietnamese Ministry of Health. Standard prevention procedure instructions in health facilities; 2012 [cited 2015 Jul 1]. Available from: www.kcb.vn/wp-content/uploads/2015/07/3.huong-dan-phong-ngua-chuan.pdf (Vietnamese).

15. Tenna A, Stenehjem EA, Margoles L, Kacha E, Blumberg HM, Kempker RR. Infection control knowledge, attitudes, and practices among healthcare workers in Addis Ababa, Ethiopia. Infect Control Hosp Epidemiol 2013;34(12):1289-1296.

16. Maheshwari V, Kaore NC, Ramnani VK, Gupta SK, Borle A, Kaushal R. A study to assess knowledge and attitude regarding hand hygiene amongst residents and nursing staff in a tertiary health care setting of Bhopal City. J Clin Diagn Res 2014;8(8):DC04DC07.

17. Salmon S, McLaws ML. Qualitative findings from focus group discussions on hand hygiene compliance among health care workers in Vietnam. Am J Infect Control 2015;43(10):10861091.

18. Socialist Republic of Vietnam. The labor code of the Socialist Republic of Vietnam; 2012 [cited 2015 Jul 1]. Available from: http://vanban.chinhphu.vn/portal/page/portal/chinhphu/ hethongvanban?class_id = 1\&mode=detail\&document_id = 163542 (Vietnamese).

19. Oh HS. Knowledge, perceptions, and self-reported performance of hand hygiene among registered nurses at community-based hospitals in the Republic of Korea: a cross-sectional multi-center study. J Prev Med Public Health 2018;51(3):121-129.

20. Nabavi M, Alavi-Moghaddam M, Gachkar L, Moeinian M. Knowledge, attitudes, and practices study on hand hygiene among Imam Hossein Hospital's residents in 2013. Iran Red Crescent Med J 2015;17(10):e19606.

21. Ango UM, Awosan KJ, Adamu H, Salawu S, Sani MM, Ibrahim $\mathrm{AH}$. Knowledge, attitude and practice of hand hygiene among healthcare providers in semi-urban communities of Sokoto State, Nigeria. Int J Trop Dis Health 2017;26(2):1-9. 
22. Asadollahi M, Arshadi Bostanabad M, Jebraili M, Mahallei M, Seyyed Rasooli A, Abdolalipour M. Nurses' knowledge regarding hand hygiene and its individual and organizational predictors. J Caring Sci 2015;4(1):45-53.

23. Tromp M, Huis A, de Guchteneire I, van der Meer J, van Achterberg $T$, Hulscher $M$, et al. The short-term and long-term effectiveness of a multidisciplinary hand hygiene improvement program. Am J Infect Control 2012;40(8):732-736.

24. Ward DJ. The role of education in the prevention and control of infection: a review of the literature. Nurse Educ Today 2011; 31(1):9-17.

25. Hung NV, Tuyen LB. A situation on and correlates of knowledge, attitude and practice of standard precautions in some northern hospitals of Vietnam. J Pract Med 2010; 716(5):36-40 (Vietnamese).

26. Ugwu MC, Muoka O, Okezie UM, Chimezie C, John D, Ezinne Ilo-Nnabuife $E$, et al. Perceptions, attitude and knowledge of five moments of hand hygiene practices among healthcare workers in Awka Anambra Nigeria. J Infect Dis Diagn 2019; 4(2):1000126.

27. Sulaiha SA, Ling WY, Chin LJ, Eow WL, MuhamadFaiz S, Tan NC. The lack of effective hand washing practice despite high level of knowledge and awareness in medical students of clinical years. Int E J Sci Med 2010;4(2):18-26.

28. Nicol PW, Watkins RE, Donovan RJ, Wynaden D, Cadwallader H.
The power of vivid experience in hand hygiene compliance. J Hosp Infect 2009;72(1):36-42.

29. Budimir-Hussey M, Ciprietti L, Ahmed F, Tarola C, Lo A, El-Masri $M$. Exploring physician hand hygiene practices and perceptions in 2 community-based Canadian hospitals. J Patient Saf 2013;9(3):140-144.

30. Yuan CT, Dembry LM, Higa B, Fu M, Wang H, Bradley EH. Perceptions of hand hygiene practices in China. J Hosp Infect 2009;71(2):157-162.

31. Kingston L, O'Connell NH, Dunne CP. Hand hygiene-related clinical trials reported since 2010: a systematic review. J Hosp Infect 2016;92(4):309-320.

32. Pan SC, Tien KL, Hung IC, Lin YJ, Yang YL, Yang MC, et al. Patient empowerment in a hand hygiene program: differing points of view between patients/family members and health care workers in Asian culture. Am J Infect Control 2013;41(11): 979-983.

33. Ofek Shlomai N, Rao S, Patole S. Efficacy of interventions to improve hand hygiene compliance in neonatal units: a systematic review and meta-analysis. Eur J Clin Microbiol Infect Dis 2015;34(5):887-897.

34. Salmon S, Truong AT, Nguyen VH, Pittet D, McLaws ML. Health care workers' hand contamination levels and antibacterial efficacy of different hand hygiene methods used in a Vietnamese hospital. Am J Infect Control 2014;42(2):178-181. 